\title{
Eigenvalues of the Laplace operator with potential under the backward Ricci flow on locally homogeneous 3-manifolds
}

\author{
Songbo Hou \\ Department of Applied Mathematics, College of Science, China Agricultural University, Beijing, 100083, P.R. China \\ Shusen Yang \\ Department of Applied Mathematics, College of Science, China Agricultural University, Beijing, 100083, P.R. China
}

\begin{abstract}
Let $\lambda(t)$ be the first eigenvalue of $-\Delta+a R(a>0)$ under the backward Ricci flow on locally homogeneous 3-manifolds, where $R$ is the scalar curvature. In the Bianchi case, we get the upper and lower bounds of $\lambda(t)$. In particular, we show that when the the backward Ricci flow converges to a sub-Riemannian geometry after a proper re-scaling, $\lambda^{+}(t)$ approaches zero, where $\lambda^{+}(t)=\max \{\lambda(t), 0\}$.
\end{abstract}

Keywords: Eigenvalue, Backward Ricci flow, Locally homogeneous 3-manifold 2020 MSC: 53E20, 58C40

\section{Introduction}

The research on the eigenvalues of operators under geometric flows has attracted many attentions. In a seminal paper [29], Perelman depicted the nondecreasing behavior of the first eigenvalue of $-\Delta+R / 4$ under the Ricci flow, where $-\Delta$ denotes the Laplace-Beltrami operator, $R$ denotes the scalar curvature. As an application, he proved the non-existence of nontrivial steady or expanding breathers on closed manifolds. Perelman's results were extended by Cao [2] to the operator $-\Delta+\frac{R}{2}$ on manifolds with nonnegative curvature operator under the Ricci flow. Li [24] removed Cao's curvature assumption and got the similar conclusion. One may refer to [3, 5, 27] for more studies about the operator $-\Delta+a R(a \geq 0)$ and refer to [32, 31] for the $p$-Laplace operator. In particular, the upper and lower bounds of eigenvalues were obtained by analyzing the evolution equation on closed Riemann surfaces [5, 32].

There are also many results under other geometric flows. In [25], Li obtained the monotonicity of eigenvalues under various re-scaled versions of Ricci flow. For the normalized powers of the $m$ th mean curvature flow, Zhao [33] established the monotonicity of the first eigenvalue of $p$-Laplace operator under certain conditions. Under the harmonic-Ricci flow, Li [26] studied the the eigenvalues and entropies. Fang and Yang [11] investigated the first eigenvalue of the operator $-\Delta_{\phi}+\frac{R}{2}$, where $-\Delta_{\phi}$ is the Witten-Laplacian and constructed monotonic quantities under

Email addresses: housb@cau.edu.cn (Songbo Hou), 13651293036@163.com (Shusen Yang) 
the Yamabe flow. Along the re-scaled List's extended Ricci flow, Huang and Li [19] considered monotonicity formulae of eigenvalues of the Laplacian and entropies. For the monotonicity of eigenvalues and quantities along the Ricci-Bourguignon flow, one may refer to [9, 30]. Related results also include $[1,7,10,12,13,15,28]$.

In general, it's difficult to get the upper and lower bounds of eigenvalues of geometric operators under flows. The obstacle is that the metric is variable, hence the classical methods, such as gradient estimates of eigenfunctions, heat kernel estimates et al., can not be employed directly. While on closed surfaces, by controlling the scalar curvature, we studied the derivative of the eigenvalue, then got the upper and lower bounds of eigenvalues by integration [5]. But the approach is not available for high dimensional manifolds. In order to explore possibilities on locally homogeneous 3-manifolds, we developed a new method to estimate the eigenvalues by comparing components of the Ricci curvature [16, 17]. Similar methods were also taken by Korouki and Razavi [23] to study the eigenvalue of $-\Delta-R$ under the Ricci flow. In this paper, we consider the eigenvalues of the operator $-\Delta+a R$ under the backward Ricci flow on locally homogeneous 3-manifolds, where $a$ is a positive constant.

There are nine classes of locally homogeneous 3-manifolds. These classes can be divided into two families. According the classification in [20], $H(3), H(2) \times \mathbb{R}$ and $\mathrm{SO}(3) \times \mathbb{R}$, where $H(n)$ means the group of isometries of hyperbolic $n$-space, belong to the first family. Six other classes $\mathbb{R}^{3}$, SU(2), SL(2, R), Heisenberg, $E(1,1)$ (the group of isometries of the plane with flat Lorentz metric) and $E(2)$ (the group of isometries of the Euclidian plane) belong to the second family which is called the Bianchi case.

In the Bianchi case, there is a Milnor frame $\left(X_{1}, X_{2}, X_{3}\right)$ which can diagonalize the initial metric and the Ricci tensor. The property that the Ricci flow keeps the diagonalization enables us to write

$$
g=A(t) \theta^{1} \otimes \theta^{1}+B(t) \theta^{2} \otimes \theta^{2}+C(t) \theta^{3} \otimes \theta^{3},
$$

where $\left(\theta^{1}, \theta^{2}, \theta^{3}\right)$ is the frame dual to $\left(X_{1}, X_{2}, X_{3}\right)$. Hence we reduce the Ricci flow to an ODE system in $(A, B, C)$. The following backward Ricci flow

$$
\frac{\partial g}{\partial t}=2 \mathrm{Rc}-\frac{2 r}{3} g, g(0)=g_{0}
$$

was studied in [4, 6]. An interesting phenomenon is that after a proper re-scaling, the flow converges uniformly to a sub-Remannian geometry in many cases. For more results about the Ricci flow on locally homogeneous manifolds, we refer the reader to [18, 21, 22] and so on.

We obtain the following theorem which extends the results in [16].

Theorem 1. Let $(M, g(t)), t \in\left[0, T_{+}\right)$, be a solution to the backward Ricci flow in the Bianchi case where $T_{+}$is the maximal existence time. Let $\lambda(t)$ be the first eigenvalue of $-\Delta+a R, a \geq 0$. When the flow converges to a sub-Riemannian geometry after a proper re-scaling, $\lambda^{+}(t)$ goes to zero as $t \rightarrow T_{+}$, where $\lambda^{+}(t)=\max \{\lambda(t), 0\}$.

Since the first eigenvalue of the Laplacian is nonnegative and the convergence is described in [16], we only study the eigenvalue of the operator $-\Delta+a R, a>0$. The remaining part of this paper is arranged as follows. In Section 2, we introduce an evolution equation of the eigenvalue under the backward Ricci flow. Since the analysis on $\mathbb{R}^{3}$ is trivial, from Section 3 to Section 7, we only investigate the behaviors of eigenvalues on Heisenberg, $\operatorname{SU}(2), E(1,1), E(2)$ and $\operatorname{SL}(2, \mathbb{R})$ case by case. 


\section{Evolution equation}

In this section, we give an evolution equation of the eigenvalue which is important in the subsequent analysis.

Theorem 2. Let $(M, g(t)), t \in\left[0, T_{+}\right)$be a solution to the backward Ricci flow on a locally homogeneous 3-manifold. Denote by $\lambda(t)$ the first eigenvalue of $-\Delta+a R, a>0$ and by $u(x, t)$ the associated positive eigenfunction with $\int u^{2}(x, t) d \mu=1$. Then under the the backward Ricci flow, there holds

$$
\frac{d}{d t} \lambda=\frac{2}{3} R \lambda-2 a|\mathrm{Rc}|^{2}-\int\left(2 R_{i j} \nabla_{i} u \nabla_{j} u\right) d \mu
$$

We omit the proof as it is similar to Lemma 3.1 in [5].

\section{Heisenberg}

For a given metric $g_{0}$, there exists a Milnor frame such that

$$
\left[X_{2}, X_{3}\right]=2 X_{1},\left[X_{3}, X_{1}\right]=0,\left[X_{1}, X_{2}\right]=0 .
$$

Let $A_{0}, B_{0}, C_{0}$ be the initial value of $A, B, C$ respectively. We take the normalization $A_{0} B_{0} C_{0}=4$. Following the calculations on page 171 in [8], one has

$$
R_{11}=\frac{1}{2} A^{3}, R_{22}=-\frac{1}{2} A^{2} B, R_{33}=-\frac{1}{2} A^{2} C, R=-\frac{1}{2} A^{2} .
$$

Then the backward Ricci flow equations become

$$
\left\{\begin{array}{l}
\frac{d}{d t} A=\frac{4}{3} A^{3} \\
\frac{d}{d t} B=-\frac{2}{3} A^{2} B \\
\frac{d}{d t} C=-\frac{2}{3} A^{2} C
\end{array}\right.
$$

Solving these equations gives

$$
\left\{\begin{array}{l}
A=A_{0}\left(1+\frac{16}{3} R_{0} t\right)^{-1 / 2} \\
B=B_{0}\left(1+\frac{16}{3} R_{0} t\right)^{1 / 4} \\
C=C_{0}\left(1+\frac{16}{3} R_{0} t\right)^{1 / 4}
\end{array}\right.
$$

where $R_{0}=-\frac{1}{2} A_{0}^{2}$. The re-scaled flow $\bar{g}(t)=\left(C_{0} / C(t)\right) g(t)$ converges uniformly to a subRiemannian geometry.

Hereafter we denote by $\tau, c_{i}$ variable constants which can be understood from the context. 
Theorem 3. Let $\lambda(t)$ be the first eigenvalue of $-\Delta+a R$. Suppose $B_{0} \geq C_{0}$. Then we get

$$
\lambda(\tau)+\frac{c_{1} A_{0}^{3}}{4\left(1-\frac{8 A_{0}^{2}}{3} \tau\right)^{3 / 2}}-\frac{c_{1} A_{0}^{3}}{4\left(1-\frac{8 A_{0}^{2}}{3} t\right)^{3 / 2}} \leq \lambda(t) \leq \lambda(\tau)\left(\frac{1-\frac{8 A_{0}^{2}}{3} t}{1-\frac{8 A_{0}^{2}}{3} \tau}\right)^{\frac{1}{8}} e^{\frac{3 B_{0}}{2}\left[\left(1-\frac{8 A_{0}^{2} \tau}{3}\right)^{1 / 4}-\left(1-\frac{8 A_{0}^{2} t}{3}\right)^{1 / 4}\right]}
$$

for $t \geq \tau$, where $\tau$ is a fixed time and $c_{1}$ is a positive constant. As a consequence, $\lambda^{+}(t) \rightarrow 0$ as $t \rightarrow 3 /\left(8 A_{0}^{2}\right)$.

Proof. Suppose $B_{0} \geq C_{0}$. we get $|\mathrm{Rc}|^{2}=\frac{3}{4} A^{4}$ by (3.1). Then 2.1) together with (3.1) implies

$$
\frac{2}{3} R \lambda-2 R_{11} \lambda-\frac{3 a}{2} A^{4}+2 a R_{11} R \leq \frac{d}{d t} \lambda \leq \frac{2}{3} R \lambda-2 R_{22} \lambda-\frac{3 a}{2} A^{4}+2 a R_{22} R .
$$

Since $A \rightarrow+\infty, B \rightarrow 0$ and $C \rightarrow 0$ as $t \rightarrow \frac{3}{8 A_{0}^{2}}$, we have

$$
-\frac{3 a}{2} A^{4}+2 a R_{22} R=-\frac{3 a}{2} A^{4}+\frac{a}{2} A^{4} B<0
$$

after a time $\tau$. This leads to

$$
\frac{d}{d t} \lambda \leq \frac{2}{3} R \lambda-2 R_{22} \lambda=\left(-\frac{1}{3} A^{2}+A^{2} B\right) \lambda
$$

for $t \geq \tau$. Hence

$$
\frac{d}{d t}\left(\lambda e^{\int_{\tau}^{t}\left(\frac{1}{3} A^{2}-A^{2} B\right) d t}\right) \leq 0
$$

It follows from the integration that

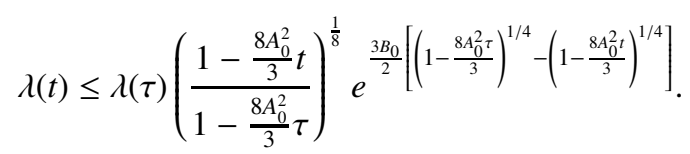

According to (3.1), (3.2), (3.3) and (3.4), we get

$$
\frac{d}{d t} \lambda \geq-c_{1} A^{5}
$$

Integration on both sides of the above inequality from $\tau$ to $t$ gives

$$
\lambda(t) \geq \lambda(\tau)+\frac{c_{1} A_{0}^{3}}{4\left(1-\frac{8 A_{0}^{2}}{3} \tau\right)^{3 / 2}}-\frac{c_{1} A_{0}^{3}}{4\left(1-\frac{8 A_{0}^{2}}{3} t\right)^{3 / 2}} .
$$

It can be easily checked that $\lambda^{+}(t)=\max \{\lambda(t), 0\}$ goes to 0 as $t$ goes to $\frac{3}{8 A_{0}^{2}}$. This finishes the proof. 


\section{4. $\mathrm{SU}(2)$}

There exists a Milnor frame for a given metric $g_{0}$ such that

$$
\left[X_{2}, X_{3}\right]=2 X_{1},\left[X_{3}, X_{1}\right]=2 X_{2},\left[X_{1}, X_{2}\right]=2 X_{3} \text {. }
$$

Under the normalization $A_{0} B_{0} C_{0}=4$, we have

$$
R_{11}=\frac{1}{2} A\left[A^{2}-(B-C)^{2}\right], R_{22}=\frac{1}{2} B\left[B^{2}-(A-C)^{2}\right], R_{33}=\frac{1}{2} C\left[C^{2}-(A-B)^{2}\right]
$$

and

$$
R=\frac{1}{2}\left[A^{2}-(B-C)^{2}\right]+\frac{1}{2}\left[B^{2}-(A-C)^{2}\right]+\frac{1}{2}\left[C^{2}-(A-B)^{2}\right] .
$$

We recall Cao's results under the assumption $A_{0} \geq B_{0} \geq C_{0}$ in [6] .

\section{Theorem 4.}

(1) If $A_{0}=B_{0}=C_{0}$, there holds $T_{+}=\infty$ and $g(t)=g_{0}$.

(2) If $A_{0}=B_{0}>C_{0}$, there holds $T_{+}=\infty, A=B>C$ and

$$
A \sim \frac{8}{3} t, \quad C \sim \frac{9}{16} t^{-2}
$$

as $t$ goes to $T_{+}$

(3) If $A_{0}>B_{0} \geq C_{0}$, there holds $T_{+}<\infty, A>B \geq C$ and

$$
A \sim \frac{\sqrt{6}}{4}\left(T_{+}-t\right)^{-1 / 2}, B \sim \eta_{1}\left(T_{+}-t\right)^{1 / 4}, C \sim \eta_{2}\left(T_{+}-t\right)^{1 / 4},
$$

where $\eta_{1}, \eta_{2}$ are two positive constants.

In the third case, $\bar{g}(t)=\left(B_{0} / B(t)\right) g(t)$ converges to a sub-Riemannian geometry.

We get the following results.

Theorem 5. Let $\lambda(t)$ be the first eigenvalue of $-\Delta+a R$. We have the following results.

(1) If $A_{0}=B_{0}=C_{0}$, then $\lambda(t)=\lambda(0)$.

(2) If $A_{0}=B_{0}>C_{0}$, then there holds

$$
\lambda(\tau)+\frac{c_{2} \lambda(\tau)}{\left(1+c_{1}\right) \tau^{c_{1}}}\left(\tau^{1+c_{1}}-t^{1+c_{1}}\right) \leq \lambda(t) \leq \lambda(\tau)\left(\frac{t}{\tau}\right)^{c_{1}}
$$

if $t \geq \tau$, where $\tau$ is a fixed time, and $c_{1}, c_{2}$ are positive constants.

(3) If $A_{0}>B_{0} \geq C_{0}$, then there holds

$$
\lambda(\tau)+c_{2}\left[\left(T_{+}-\tau\right)^{-3 / 2}-\left(T_{+}-t\right)^{-3 / 2}\right] \leq \lambda(t) \leq \lambda(\tau)\left(\frac{T_{+}-t}{T_{+}-\tau}\right)^{c_{1}}
$$

if $t \geq \tau$, where $\tau$ is a fixed time, and $c_{1}, c_{2}$ are positive constants. In this case, $\lambda^{+}(t) \rightarrow 0$ as $t \rightarrow T_{+}$. 
Proof.

(1) If $A_{0}=B_{0}=C_{0}$, then $\lambda(t)$ is a constant.

(2) If $A_{0}=B_{0}>C_{0}$, Theorem 4.2 in [16] implies

$$
R_{11}=R_{22}>R_{33}>0
$$

after a time $\tau$. Then it follows that

$$
\frac{2}{3} R \lambda-2 R_{11} \lambda-2 a|\mathrm{Rc}|^{2}+2 a R_{11} R \leq \frac{d}{d t} \lambda \leq \frac{2}{3} R \lambda-2 R_{33} \lambda-2 a|\mathrm{Rc}|^{2}+2 a R_{33} R .
$$

By employing (4.1), (4.2) and the second item in Theorem 4, we have

$$
\begin{gathered}
2 a|\mathrm{Rc}|^{2}=\frac{a}{2}\left[\left(2 B C-C^{2}\right)^{2}+\left(2 B C-C^{2}\right)^{2}+C^{4}\right] \sim c_{1} t^{-2}, \\
2 a R_{11} R=\frac{a A}{2}\left(2 B C-C^{2}\right)\left[2 B C-C^{2}+2 A C-C^{2}+C^{2}\right] \sim c_{2} t^{-1}, \\
\frac{2}{3} R \lambda-2 R_{11} \lambda=\left(\frac{4}{3} B C-\frac{1}{3} C^{2}-2 A B C+A C^{2}\right) \lambda \sim-8 \lambda, \\
\frac{2}{3} R \lambda-2 R_{33} \lambda=\left(\frac{4}{3} B C-\frac{1}{3} C^{2}-C^{3}\right) \lambda \sim 2 t^{-1} \lambda, \\
2 a R_{33} R \sim c_{3} t^{-7} .
\end{gathered}
$$

Hence by (4.3), (4.4) and (4.6), we get

$$
\frac{d}{d t} \lambda \leq\left(\frac{4}{3} B C-\frac{1}{3} C^{2}-C^{3}\right) \lambda .
$$

Denoting $D=\frac{4}{3} B C-\frac{1}{3} C^{2}-C^{3}$, we obtain

$$
\frac{d}{d t}\left(\lambda e^{\int_{\tau}^{t}-D d t}\right) \leq 0
$$

if $t \geq \tau$. It is easy to see that

$$
\lambda(t) \leq \lambda(\tau)\left(\frac{t}{\tau}\right)^{c_{1}}
$$

Hence (4.3) together with 4.4, 4.5) and 4.7) leads to

$$
-c_{2} \lambda(\tau)\left(\frac{t}{\tau}\right)^{c_{1}} \leq\left(\frac{4}{3} B C-\frac{1}{3} C^{2}-2 A B C+A C^{2}\right) \lambda(\tau)\left(\frac{t}{\tau}\right)^{c_{1}} \leq \frac{d}{d t} \lambda .
$$

Integration from $\tau$ to $t$ gives

$$
\lambda(\tau)+\frac{c_{2} \lambda(\tau)}{\left(1+c_{1}\right) \tau^{c_{1}}}\left(\tau^{1+c_{1}}-t^{1+c_{1}}\right) \leq \lambda(t)
$$

(3) If $A_{0}>B_{0} \geq C_{0}$, it follows from Theorem 4.2 in [16] that

$$
R_{11}>0, R_{22}<0, R_{33}<0
$$


and

$$
R_{11}>R_{33} \geq R_{22}
$$

after a time $\tau$. Hence

$$
\frac{2}{3} R \lambda-2 R_{11} \lambda-2 a|\mathrm{Rc}|^{2}+2 a R_{11} R \leq \frac{d}{d t} \lambda \leq \frac{2}{3} R \lambda-2 R_{22} \lambda-2 a|\mathrm{Rc}|^{2}+2 a R_{22} R .
$$

Using (4.1), (4.2) and the third item in Theorem 4, we get

$$
\begin{gathered}
2 a|\mathrm{Rc}|^{2}=\frac{a}{2}\left\{\left[A^{2}-(B-C)^{2}\right]^{2}+\left[B^{2}-(A-C)^{2}\right]^{2}+\left[C^{2}-(A-B)^{2}\right]^{2}\right\} \sim c_{1}\left(T_{+}-t\right)^{-2}, \\
2 a R_{11} R=\frac{a A}{2}\left[A^{2}-(B-C)^{2}\right]\left(2 A B+2 A C+2 B C-A^{2}-B^{2}-C^{2}\right) \sim-c_{2}\left(T_{+}-t\right)^{-5 / 2}, \\
\frac{2}{3} R \lambda-2 R_{11} \lambda \sim-c_{3}\left(T_{+}-t\right)^{-3 / 2} \lambda, \\
\frac{2}{3} R \lambda-2 R_{22} \lambda \sim-c_{4}\left(T_{+}-t\right)^{-1} \lambda, \\
2 a R_{22} R \sim c_{5}\left(T_{+}-t\right)^{-7 / 4} .
\end{gathered}
$$

By (4.8), (4.9) and (4.11), we obtain

$$
\frac{d}{d t} \lambda \leq \frac{2}{3} R \lambda-2 R_{22} \lambda
$$

if $t \geq \tau$. Integrating from $\tau$ to $t$, we have

$$
\lambda(t) \leq \lambda(\tau) e^{\int_{\tau}^{t}\left(\frac{2}{3} R-2 R_{22}\right) d t} \leq \lambda(\tau)\left(\frac{T_{+}-t}{T_{+}-\tau}\right)^{c_{1}},
$$

which together with (4.8), (4.9) and (4.10) implies

$$
-c_{2}\left(T_{+}-t\right)^{-5 / 2} \leq \frac{d}{d t} \lambda
$$

By integration, we obtain

$$
\lambda(\tau)+c_{2}\left[\left(T_{+}-\tau\right)^{-3 / 2}-\left(T_{+}-t\right)^{-3 / 2}\right] \leq \lambda(t) .
$$

Finally, it is true that $\lambda^{+}(t) \rightarrow 0$ as $t \rightarrow T_{+}$.

\section{5. $E(1,1)$}

Given a metric $g_{0}$, we have a fixed Milnor frame such that

$$
\left[X_{2}, X_{3}\right]=2 X_{1},\left[X_{3}, X_{1}\right]=0 \quad\left[X_{1}, X_{2}\right]=-2 X_{3} .
$$

Choosing the normalization $A_{0} B_{0} C_{0}=4$, one has

$$
R_{11}=\frac{1}{2} A\left(A^{2}-C^{2}\right), R_{22}=-\frac{1}{2} B(A+C)^{2}, R_{33}=\frac{1}{2} C\left(C^{2}-A^{2}\right), R=-\frac{1}{2}(A+C)^{2} .
$$

Suppose $A_{0} \geq C_{0}$. Cao [6] described the following behaviors. 


\section{Theorem 6.}

(1) If $A_{0}=C_{0}$, then we have $T_{+}=\frac{3}{32} B_{0}$ and

$$
A(t)=C(t)=\frac{\sqrt{6}}{4}\left(T_{+}-t\right)^{-1 / 2}, \quad B(t)=\frac{32}{3}\left(T_{+}-t\right), \quad t \in\left[0, T_{+}\right) .
$$

(2) If $A_{0}>C_{0}$, then we have $T_{+}<\infty$, and

$$
A \sim \frac{\sqrt{6}}{4}\left(T_{+}-t\right)^{-1 / 2}, B(t) \sim \eta_{1}\left(T_{+}-t\right)^{1 / 4}, C(t) \sim \eta_{2}\left(T_{+}-t\right)^{1 / 4},
$$

as $t$ goes to $T_{+}$, where $\eta_{1}, \eta_{2}$ are two positive constants.

In the second case, $\bar{g}(t)=\left(B_{0} / B(t)\right) g(t)$ converges to a sub-Riemannian geometry.

We investigate the eigenvalue and get the following results.

Theorem 7. Let $\lambda(t)$ be the first eigenvalue of $-\Delta+a R$. Then we get (1) If $A_{0}=C_{0}$, then we have

$$
\lambda(\tau)+c_{1}\left[\left(T_{+}-\tau\right)^{-1}-\left(T_{+}-t\right)^{-1}\right] \leq \lambda(t) \leq \lambda(\tau)\left(\frac{T_{+}-t}{T_{+}-\tau}\right)^{1 / 2} e^{16(t-\tau)}
$$

if $t \geq \tau$, where $\tau$ is a fixed time and $c_{1}$ is a positive constant.

(2) If $A_{0}>C_{0} \geq B_{0}$, then for $t \geq \tau$, there holds

$$
\lambda(\tau)+c_{2}\left[\left(T_{+}-\tau\right)^{-\frac{3}{2}}-\left(T_{+}-t\right)^{-\frac{3}{2}}\right] \leq \lambda(t) \leq \lambda(\tau)\left(\frac{T_{+}-t}{T_{+}-\tau}\right)^{c_{1}} .
$$

In both cases, $\lambda^{+}(t) \rightarrow 0$ as $t \rightarrow T_{+}$.

Remark. If $A_{0}>B_{0}>C_{0}$, the analogous estimates hold.

Proof. (1) If $A_{0}=C_{0}$, then (5.1) together with the first item in Theorem 6 implies

$$
R_{11}=0, R_{22}<0, R_{33}=0
$$

and

$$
R_{11}=R_{33}>R_{22}
$$

Then we have

$$
\frac{2}{3} R \lambda-2 a|\mathrm{Rc}|^{2} \leq \frac{d}{d t} \lambda \leq \frac{2}{3} R \lambda-2 R_{22} \lambda-2 a|\mathrm{Rc}|^{2}+2 a R_{22} R
$$

There exist a time $\tau$ such that

$$
\begin{gathered}
2 a|\mathrm{Rc}|^{2}=\frac{a}{2}\left[\left(A^{2}-C^{2}\right)^{2}+(A+C)^{4}+\left(C^{2}-A^{2}\right)^{2}\right] \sim c_{1}\left(T_{+}-t\right)^{-2}, \\
2 a R_{22} R=\frac{a}{2} B(A+C)^{4} \sim c_{2}\left(T_{+}-t\right)^{-1}
\end{gathered}
$$

if $t \geq \tau$. Hence it follows from (5.2), (5.3) and (5.4) that

$$
\frac{d}{d t} \lambda \leq \frac{2}{3} R \lambda-2 R_{22} \lambda=\left(-\frac{1}{2}\left(T_{+}-t\right)^{-1}+16\right) \lambda
$$


for $t \geq \tau$. This leads to

$$
\frac{d}{d t}\left(\lambda e^{\int_{\tau}^{t}\left(\frac{1}{2}\left(T_{+}-t\right)^{-1}-16\right) d t}\right) \leq 0
$$

Integration from $\tau$ to $t$ gives

$$
\lambda(t) \leq \lambda(\tau)\left(\frac{T_{+}-t}{T_{+}-\tau}\right)^{1 / 2} e^{16(t-\tau)} .
$$

By (5.2), (5.3) and (5.5), we get

$$
-c_{1}\left(T_{+}-t\right)^{-2} \leq \frac{d}{d t} \lambda
$$

after a time $\tau$. It is concluded by integration that

$$
\lambda(\tau)+c_{1}\left[\left(T_{+}-\tau\right)^{-1}-\left(T_{+}-t\right)^{-1}\right] \leq \lambda(t) .
$$

(2) If $A_{0}>C_{0} \geq B_{0}$, by Theorem 5.2 in [16] we have

$$
R_{11}>0, R_{22}<0, R_{33}<0
$$

and

after a time $\tau$.

$$
R_{11}>R_{22}>R_{33}
$$

It follows that

$$
\frac{2}{3} R \lambda-2 R_{11} \lambda-2 a|\mathrm{Rc}|^{2}+2 a R_{11} R \leq \frac{d}{d t} \lambda \leq \frac{2}{3} R \lambda-2 R_{33} \lambda-2 a|\mathrm{Rc}|^{2}+2 a R_{33} R .
$$

Combining (5.1) and the second item in Theorem 6, we calculate

$$
\begin{gathered}
2 a|\mathrm{Rc}|^{2}=\frac{a}{2}\left[\left(A^{2}-C^{2}\right)^{2}+(A+C)^{4}+\left(C^{2}-A^{2}\right)^{2}\right] \sim c_{1}\left(T_{+}-t\right)^{-2}, \\
2 a R_{33} R=\frac{a}{2} C(A+C)^{2}\left(A^{2}-C^{2}\right) \sim c_{2}\left(T_{+}-t\right)^{-\frac{7}{4}}, \\
2 a R_{11} R=-\frac{a}{2} A\left(A^{2}-C^{2}\right)(A+C)^{2} \sim-c_{3}\left(T_{+}-t\right)^{-\frac{5}{2}} .
\end{gathered}
$$

By (5.6), (5.7) and (5.8), we obtain

$$
\frac{d}{d t} \lambda \leq \frac{2}{3} R \lambda-2 R_{33} \lambda
$$

for $t \geq \tau$.

Integration from $\tau$ to $t$ yields

$$
\lambda(t) \leq \lambda(\tau) e^{\int_{\tau}^{t}\left(\frac{2}{3} R-2 R_{33}\right) d t} \leq \lambda(\tau)\left(\frac{T_{+}-t}{T_{+}-\tau}\right)^{c_{1}} .
$$

It is clear that $\lambda^{+}(t) \rightarrow 0$ as $t \rightarrow T_{+}$. Using (5.6), (5.7), (5.9) and (5.10), we immediately get

It follows from the integration that

$$
-c_{2}\left(T_{+}-t\right)^{-\frac{5}{2}} \leq \frac{d}{d t} \lambda
$$

$$
\lambda(\tau)+c_{2}\left[\left(T_{+}-\tau\right)^{-\frac{3}{2}}-\left(T_{+}-t\right)^{-\frac{3}{2}}\right] \leq \lambda(t) .
$$


6. $E(2)$

There is a Milnor frame for a metric $g_{0}$ such that

$$
\left[X_{2}, X_{3}\right]=2 X_{1},\left[X_{3}, X_{1}\right]=2 X_{2},\left[X_{1}, X_{2}\right]=0 .
$$

Choosing the normalization $A_{0} B_{0} C_{0}=4$, then we have

$$
R_{11}=\frac{1}{2} A\left(A^{2}-B^{2}\right), R_{22}=\frac{1}{2} B\left(B^{2}-A^{2}\right), R_{33}=-\frac{1}{2} C(A-B)^{2}, R=-\frac{1}{2}(A-B)^{2} .
$$

Cao [6] gave the following results.

\section{Theorem 8.}

(1) If $A_{0}=B_{0}$, there holds $T_{+}=\infty$, and $g(t)=g_{0}$.

(2) If $A_{0}>B_{0}$, then there holds $T_{+}<\infty$, and

$$
A \sim \frac{\sqrt{6}}{4}\left(T_{+}-t\right)^{-1 / 2}, B(t) \sim \eta_{1}\left(T_{+}-t\right)^{1 / 4}, C(t) \sim \eta_{2}\left(T_{+}-t\right)^{1 / 4}
$$

as $t$ goes to $T_{+}$, where $\eta_{1}, \eta_{2}$ are two positive constants.

In the second case, $\bar{g}(t)=\left(B_{0} / B(t)\right) g(t)$ converges to a sub-Riemannian geometry.

We will prove the following theorem.

Theorem 9. Let $\lambda(t)$ be the first eigenvalue of $-\Delta+a R$. Then we get

(1) If $A_{0}=B_{0}$, then $g(t)=g_{0}$, and $\lambda(t)=\lambda(0)$.

(2) If $A_{0}>B_{0}$ and $C_{0} \geq B_{0}$, there holds that

$$
\lambda(\tau)+c_{2}\left[\left(T_{+}-\tau\right)^{-\frac{3}{2}}-\left(T_{+}-t\right)^{-\frac{3}{2}}\right] \leq \lambda(t) \leq \lambda(\tau)\left(\frac{T_{+}-t}{T_{+}-\tau}\right)^{c_{1}}
$$

for $t \geq \tau$. In this case, $\lambda^{+}(t) \rightarrow 0$ as $t \rightarrow T_{+}$

Remark. If $A_{0}>B_{0}$ and $C_{0}<B_{0}$, the similar estimates hold.

Proof.

(1) If $A_{0}=B_{0}$, then $g(t)=g_{0}$, and $\lambda(t)=\lambda(0)$.

(2) If $A_{0}>B_{0}$, by Theorem 6.2 in [16], we know that

$$
R_{11}>0, R_{22}<0, R_{33}<0
$$

and $R_{11}>R_{22}>R_{33}$ with $t \geq \tau$ for some $\tau$.

It is easy to see that

$$
\frac{2}{3} R \lambda-2 R_{11} \lambda-2 a|\mathrm{Rc}|^{2}+2 a R_{11} R \leq \frac{d}{d t} \lambda \leq \frac{2}{3} R \lambda-2 R_{33} \lambda-2 a|\mathrm{Rc}|^{2}+2 a R_{33} R
$$

for $t \geq \tau$.

By (6.1) and the second item in Theorem 8, we arrive at

$$
2 a|\mathrm{Rc}|^{2}=\frac{a}{2}\left[\left(A^{2}-B^{2}\right)^{2}+\left(B^{2}-A^{2}\right)^{2}+(A-B)^{4}\right] \sim c_{1}\left(T_{+}-t\right)^{-2},
$$




$$
\begin{gathered}
2 a R_{33} R=\frac{a}{2} C(A-B)^{4} \sim c_{2}\left(T_{+}-t\right)^{-7 / 4}, \\
2 a R_{11} R=-\frac{a}{2} A\left(A^{2}-B^{2}\right)(A-B)^{2} \sim-c_{3}\left(T_{+}-t\right)^{-5 / 2} .
\end{gathered}
$$

Hence (6.2) together with (6.3) and (6.4) leads to

$$
\frac{d}{d t} \lambda \leq \frac{2}{3} R \lambda-2 R_{33} \lambda
$$

By integration from $\tau$ to $t$, we get

$$
\lambda(t) \leq \lambda(\tau) e^{\int_{\tau}^{t}\left(\frac{2}{3} R-2 R_{33}\right) d t} \leq \lambda(\tau)\left(\frac{T_{+}-t}{T_{+}-\tau}\right)^{c_{1}},
$$

which implies that $\lambda^{+}(t) \rightarrow 0$ as $t \rightarrow T_{+}$. Observing (6.2), (6.3), (6.5) and (6.6), we obtain

$$
-c_{2}\left(T_{+}-t\right)^{-\frac{5}{2}} \leq \frac{d}{d t} \lambda
$$

We conclude by the integration that

$$
\lambda(\tau)+c_{2}\left[\left(T_{+}-\tau\right)^{-\frac{3}{2}}-\left(T_{+}-t\right)^{-\frac{3}{2}}\right] \leq \lambda(t) .
$$

\section{7. $\operatorname{SL}(2, \mathbb{R})$}

This class is characterized by the Lie bracket of the Milnor frame:

$$
\left[X_{2}, X_{3}\right]=-2 X_{1}, \quad\left[X_{3}, X_{1}\right]=2 X_{2}, \quad\left[X_{1}, X_{2}\right]=2 X_{3} .
$$

Under the normalization $A_{0} B_{0} C_{0}=4$, we have

$$
R_{11}=\frac{1}{2} A\left[A^{2}-(B-C)^{2}\right], R_{22}=\frac{1}{2} B\left[B^{2}-(A+C)^{2}\right], R_{33}=\frac{1}{2} C\left[C^{2}-(A+B)^{2}\right]
$$

and

$$
R=\frac{1}{2}\left[A^{2}-(B-C)^{2}\right]+\frac{1}{2}\left[B^{2}-(A+C)^{2}\right]+\frac{1}{2}\left[C^{2}-(A+B)^{2}\right] .
$$

Under the assumption $B_{0} \geq C_{0}$, Cao [4, 6] proved the following theorem.

Theorem 10. The backward Ricci flow exists in a finite time and has the following asymptotic behaviors:

(1) If there exists a time $t_{0}$ such that $A\left(t_{0}\right) \geq B\left(t_{0}\right)$, there holds

$$
A \sim \frac{\sqrt{6}}{4}\left(T_{+}-t\right)^{-1 / 2}, B(t) \sim \eta_{1}\left(T_{+}-t\right)^{1 / 4}, C(t) \sim \eta_{2}\left(T_{+}-t\right)^{1 / 4},
$$

where $\eta_{1}, \eta_{2}$ are two positive constants.

(2) If there exists a time $t_{0}$ such that $A\left(t_{0}\right) \leq B\left(t_{0}\right)-C\left(t_{0}\right)$, there holds

$$
A \sim \eta_{1}\left(T_{+}-t\right)^{1 / 4}, B(t) \sim \frac{\sqrt{6}}{4}\left(T_{+}-t\right)^{-1 / 2}, C(t) \sim \eta_{2}\left(T_{+}-t\right)^{1 / 4}
$$


with positive constants $\eta_{1}$ and $\eta_{2}$.

(3) If $A<B<A+C$ for all time $t \in\left[0, T_{+}\right)$, we arrive at

$$
A \sim \frac{\sqrt{6}}{4}\left(T_{+}-t\right)^{-1 / 2}, B(t) \sim \frac{\sqrt{6}}{4}\left(T_{+}-t\right)^{-1 / 2}, C(t) \sim \frac{32}{3}\left(T_{+}-t\right) .
$$

In all cases, the metric $g(t)$ converges to a sub-Riemannian geometry after a proper rescaling.

We have the following theorem.

Theorem 11. Let $\lambda(t)$ be the first eigenvalue of $-\Delta+a R$. Then we get

(1) If there is a time $t_{0}$ such that $A\left(t_{0}\right) \geq B\left(t_{0}\right)$, then there exists a time $\tau$ such that

$$
\lambda(\tau)+c_{2}\left[\left(T_{+}-\tau\right)^{-\frac{3}{2}}-\left(T_{+}-t\right)^{-\frac{3}{2}}\right] \leq \lambda(t) \leq \lambda(\tau)\left(\frac{T_{+}-t}{T_{+}-\tau}\right)^{c_{1}}
$$

for $t \geq \tau$.

(2) If there exist a time $t_{0}$ such that $A\left(t_{0}\right) \leq B\left(t_{0}\right)-C\left(t_{0}\right)$, and a time $t_{1}$ such that $A\left(t_{1}\right)>C\left(t_{1}\right)$, then there is a time $\tau$ such that

$$
\lambda(\tau)+c_{2}\left[\left(T_{+}-\tau\right)^{-1}-\left(T_{+}-t\right)^{-1}\right] \leq \lambda(t) \leq \lambda(\tau)\left(\frac{T_{+}-t}{T_{+}-\tau}\right)^{c_{1}}
$$

for $t \geq \tau$.

(3) If $A<B<A+C$ for all time $t \in\left[0, T_{+}\right)$, then there is time $\tau$ such that

$$
\lambda(\tau)+c_{2}\left[\left(T_{+}-\tau\right)^{-1}-\left(T_{+}-t\right)^{-1}\right] \leq \lambda(t) \leq \lambda(\tau)\left(\frac{T_{+}-t}{T_{+}-\tau}\right)^{c_{1}}
$$

for $t \geq \tau$.

In all cases, $\lambda^{+}(t) \rightarrow 0$ as $t \rightarrow T_{+}$.

Remark. In the second item of the above theorem, an analogous estimate holds if $A(t) \leq C(t)$ for all $t$.

Proof. (1) If there is a time $t_{0}$ such that $A\left(t_{0}\right)>B_{0}$, then we know from Theorem 7.2 in [16] that

$$
R_{11}>0, R_{22}<0, R_{33}<0
$$

and

$$
R_{11}>R_{33} \geq R_{22}
$$

after a time $\tau$.

Hence

$$
\frac{2}{3} R \lambda-2 R_{11} \lambda-2 a|\mathrm{Rc}|^{2}+2 a R_{11} R \leq \frac{d}{d t} \lambda \leq \frac{2}{3} R \lambda-2 R_{22} \lambda-2 a|\mathrm{Rc}|^{2}+2 a R_{22} R
$$

for $t \geq \tau$. It follows from (7.1), (7.2) and the first item in Theorem 10 that

$$
2 a|\mathrm{Rc}|^{2}=\frac{a}{2}\left[A^{2}-(B-C)^{2}\right]^{2}+\frac{a}{2}\left[B^{2}-(A+C)^{2}\right]^{2}+\frac{a}{2}\left[C^{2}-(A+B)^{2}\right]^{2} \sim c_{1}\left(T_{+}-t\right)^{-2},
$$




$$
\begin{aligned}
& 2 a R_{22} R=\frac{a}{2} B\left[B^{2}-(A+C)^{2}\right]\left[2 B C-2 A C-2 A B-A^{2}-B^{2}-C^{2}\right] \sim c_{2}\left(T_{+}-t\right)^{-7 / 4} \\
& 2 a R_{11} R=\frac{a}{2} A\left[A^{2}-(B-C)^{2}\right]\left[2 B C-2 A C-2 A B-A^{2}-B^{2}-C^{2}\right] \sim-c_{3}\left(T_{+}-t\right)^{-5 / 2}
\end{aligned}
$$

Then by (7.3), (7.4) and (7.5), we arrive at

$$
\frac{d}{d t} \lambda \leq \frac{2}{3} R \lambda-2 R_{22} \lambda
$$

if $t \geq \tau$. Integration from $\tau$ to $t$ gives

$$
\lambda(t) \leq \lambda(\tau) e^{\int_{\tau}^{t}\left(\frac{2}{3} R-2 R_{22}\right) d t} \leq \lambda(\tau)\left(\frac{T_{+}-t}{T_{+}-\tau}\right)^{c_{1}} .
$$

Thus, (7.3) together with (7.4), (7.6), (7.7) implies

$$
-c_{2}\left(T_{+}-t\right)^{-\frac{5}{2}} \leq \frac{d}{d t} \lambda
$$

It is concluded by integration that

$$
\lambda(\tau)+c_{2}\left[\left(T_{+}-\tau\right)^{-\frac{3}{2}}-\left(T_{+}-t\right)^{-\frac{3}{2}}\right] \leq \lambda(t) .
$$

(2) If there exists a time $t_{0}$ such that $A\left(t_{0}\right) \leq B\left(t_{0}\right)-C\left(t_{0}\right)$ and a time $t_{1}$ such that $A\left(t_{1}\right)>C\left(t_{1}\right)$, then by Theorem 7.2 in [16], there holds

$$
R_{11}<0, R_{22}>0, R_{33}<0
$$

and

$$
R_{22}>R_{33}>R_{11}
$$

after a time $\tau$.

We obtain

$$
\frac{2}{3} R \lambda-2 R_{22} \lambda-2 a|\mathrm{Rc}|^{2}+2 a R_{22} R \leq \frac{d}{d t} \lambda \leq \frac{2}{3} R \lambda-2 R_{11} \lambda-2 a|\mathrm{Rc}|^{2}+2 a R_{11} R
$$

for $t \geq \tau$.

Calculate

$$
\begin{gathered}
2 a|\mathrm{Rc}|^{2} \sim c_{1}\left(T_{+}-t\right)^{-2}, \\
2 a R_{11} R=\frac{a}{2} A\left[A^{2}-(B-C)^{2}\right]\left[2 B C-2 A C-2 A B-A^{2}-B^{2}-C^{2}\right] \sim c_{2}\left(T_{+}-t\right)^{-7 / 4}, \\
2 a R_{22} R=\frac{a}{2} B\left[B^{2}-(A+C)^{2}\right]\left[2 B C-2 A C-2 A B-A^{2}-B^{2}-C^{2}\right] \sim-c_{3}\left(T_{+}-t\right)^{-5 / 2} .
\end{gathered}
$$

Using the similar arguments as in the first case, we get

$$
\lambda(\tau)+c_{2}\left[\left(T_{+}-\tau\right)^{-1}-\left(T_{+}-t\right)^{-1}\right] \leq \lambda(t) \leq \lambda(\tau)\left(\frac{T_{+}-t}{T_{+}-\tau}\right)^{c_{1}}
$$

for $t \geq \tau$. 
(3) If $A<B<A+C$ for all time $t \in\left[0, T_{+}\right)$, then Theorem 7.2 in [16] implies

$$
R_{11}>0, R_{22}<0, R_{33}<0
$$

and

$$
R_{11}>R_{22} \geq R_{33}
$$

after a time $\tau$. Consequently,

$$
\frac{2}{3} R \lambda-2 R_{11} \lambda-2 a|\mathrm{Rc}|^{2}+2 a R_{11} R \leq \frac{d}{d t} \lambda \leq \frac{2}{3} R \lambda-2 R_{33} \lambda-2 a|\mathrm{Rc}|^{2}+2 a R_{33} R
$$

for $t \geq \tau$.

Direct calculations give

$$
\begin{gathered}
2 a|\mathrm{Rc}|^{2} \sim c_{1}\left(T_{+}-t\right)^{-2}, \\
2 a R_{11} R=\frac{a}{2} A\left[A^{2}-(B-C)^{2}\right]\left[2 B C-2 A C-2 A B-A^{2}-B^{2}-C^{2}\right] \sim-c_{2}\left(T_{+}-t\right)^{-1}, \\
2 a R_{33} R=\frac{a}{2} C\left[C^{2}-(A+B)^{2}\right]\left[2 B C-2 A C-2 A B-A^{2}-B^{2}-C^{2}\right] \sim c_{3}\left(T_{+}-t\right)^{-7 / 4} .
\end{gathered}
$$

Proceeding as in the proof of the first case, we have

$$
\lambda(\tau)+c_{2}\left[\left(T_{+}-\tau\right)^{-1}-\left(T_{+}-t\right)^{-1}\right] \leq \lambda(t) \leq \lambda(\tau)\left(\frac{T_{+}-t}{T_{+}-\tau}\right)^{c_{1}}
$$

for $t \geq \tau$.

\section{Acknowledgement}

This work is partially supported by the National Natural Science Foundation of China (Grant No. 11721101), and by National Key Research and Development Project SQ2020YFA070080.

\section{References}

[1] Abimbola Abolarinwa, Eigenvalues of the weighted Laplacian under the extended Ricci flow, Adv. Geom. 19 (1) (2019) 131-143.

[2] Xiaodong Cao, Eigenvalues of $\left(-\Delta+\frac{R}{2}\right)$ on manifolds with nonnegative curvature operator, Math. Ann. 337 (2) (2007) 435-441.

[3] Xiaodong Cao, First eigenvalues of geometric operators under the Ricci flow, Proc. Am. Math. Soc. 136 (11) (2008) 4075-4078.

[4] Xiaodong Cao, John Guckenheimer, Laurent Saloff-Coste, The backward behavior of the Ricci and cross curvature flows on SL(2, R), Comm. Anal. Geom. 17 (4) (2009) 777-796.

[5] Xiaodong Cao, Songbo Hou, Jun Ling, Estimate and monotonicity of the first eigenvalue under the Ricci flow, Math. Ann. 354 (2012) 451-463.

[6] Xiaodong Cao, Laurent Saloff-Coste, Backward Ricci flow on locally homogeneous three-manifolds, Comm. Anal. Geom. 12 (2) (2009) 305-325.

[7] Bin Chen, Qun He, Fanqi Zeng, Monotonicity of eigenvalues of geometric operators along the Ricci-Bourguignon flow, Pacific J. Math. 296 (1) (2018) 1-20.

[8] Bennett Chow, Peng Lu, Lei Ni, Hamilton's Ricci Flow, American Mathematical Society/Science Press, 2006. 
[9] Farzad Daneshvar, Asadollah Razavi, Evolution and monotonicity for a class of quantities along the RicciBourguignon flow, J. Korean Math. Soc. 56 (6) (2019) 1441-1461.

[10] Shouwen Fang, Haifeng Xu, Peng Zhu, Evolution and monotonicity of eigenvalues under the Ricci flow, Sci. China Math. 58 (8) (2015) 1737-1744.

[11] Shouwen Fang, Fei Yang, First eigenvalues of geometric operators under the Yamabe flow, Bull. Korean Math. Soc. 53 (2016) 1113-1122.

[12] Shouwen Fang, Fei Yang, Peng Zhu, Eigenvalues of geometric operators related to the Witten Laplacian under the Ricci flow, Glasg. Math. J. 59 (3) (2017) 743-751.

[13] Hongxin Guo, Robert Philipowski, Anton Thalmaier, Entropy and lowest eigenvalue on evolving manifolds, Pacific J. Math. 264 (1) (2013) 61-81.

[14] Pak Tung Ho, First eigenvalues of geometric operators under the Yamabe flow, Ann. Global Anal. Geom. 54 (3) (2018) 449-472.

[15] Pak Tung Ho, Hyunmo Koo, Evolution of the Steklov eigenvalue under geodesic curvature flow, Manuscripta Math. 159 (2019) 453-473.

[16] Songbo Hou, Eigenvalues under the backward Ricci flow on locally homogeneous closed 3-manifolds, Acta Mathematics Sinica, English Series, 34 (7) (2018) 1179-1194.

[17] Songbo Hou, Eigenvalues under the Ricci flow of model geometries, Acta Mathematics Sinica, Chinese Series 60 (4) (2017) 583-594.

[18] Songbo Hou, Quasi-convergence of the Ricci flow on locally homogeneous closed 4-manifolds, Differential Geom. Appl. 52 (2017) 20-41.

[19] Guangyue Huang, Zhi Li, Monotonicity formulas of eigenvalues and energy functionals along the rescaled List's extended Ricci flow, Mediterr. J. Math. 15 (2) (2018) Art. 63.

[20] James Isenberg, Martin Jacken, Ricci flow of locally homogeneous geometries of closed manifolds, J. Differ. Geome. 35 (1992) 723-741.

[21] James Isenberg, Martin Jacken, Peng Lu, Ricci flow on locally homogeneous closed 4-manifolds, Comm. Anal. Geom. 14 (2) (2006) 345-386.

[22] Dan Knopf and Kevin McLeod, Quasi-convergence of model geometries under the Ricci flow, Comm. Anal. Geom. 9 (4) (2001) 879-919.

[23] F. Korouki, A. Razavi, Bounds for the first eigenvalue of $(-\Delta-R)$ under the Ricci flow on Bianchi classes, Bull. Braz. Math. Soc., New Series (2019).

[24] Junfang Li, Eigenvalues and energy functionals with monotonicity formulae under Ricci flow, Math. Ann. 338 (2007) 927-946.

[25] Junfang Li, Evolution of eigenvalues along rescaled Ricci flow, Canad. Math. Bull. Vol. 56 (1) (2013) 127-135.

[26] Yi Li, Eigenvalues and entropies under the harmonic-Ricci flow, Pacific J. Math. 267 (2014) 141-184.

[27] Li Ma, Eigenvalue monotonicity for the Ricci-Hamilton flow, Ann. Glob. Anal. Geom. 29 (3) (2006) 287-292

[28] Jing Mao, Monotonicity of the first eigenvalue of the Laplace and the $p$-Laplace operators under a forced mean curvature flow, J. Korean Math. Soc. 55 (6) (2018) 1435-1458.

[29] G. Perelman, The entropy formula for the Ricci flow and its geometric applications, arXiv:math/0211159 1,2002

[30] Linfeng Wang, Monotonicity of eigenvalues and functionals along the Ricci-Bourguignon flow, J. Geom. Anal. 29 (2) (2019) 1116-1135

[31] Jiayong Wu, First eigenvalue monotonicity for the $p$-Laplace operator under the Ricci flow, Acta Mathematica Sinica-English Series 27 (8) (2011) 1591-1598.

[32] Jiayong Wu, Ermin Wang and Yu Zheng, First eigenvalue of the $p$-Laplace operator along the Ricci flow, Ann Glob. Anal. Geom. 38 (1) (2010) 27-55

[33] Liang Zhao, The first eigenvalue of $p$-Laplace operator under powers of the $m$ th mean curvature flow, Results. Math. 63 (2013) 937-948. 